\title{
Review: the use of computed tomographic scanning has high sensitivity, specificity, and accuracy for diagnosing acute appendicitis in adults
}

Neumayer L, Kennedy A. Imaging in appendicitis: a review with special emphasis on the treatment of women. Obstet Gynecol 2003;102:1404-9.

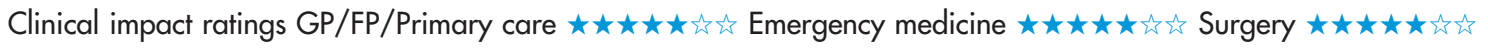

In adults, what is the accuracy of computed tomographic (CT) scanning for diagnosing appendicitis?

\section{METHODS}

Data sources: Medline (1998 to July 2003) and reference lists of
relevant articles.

\section{MAIN RESULTS}

23 studies used prospective designs to address the diagnostic accuracy of CT scanning. In these studies, the numbers of patients ranged between 31 and 308. CT features used to diagnose acute appendicitis included appendiceal diameter $>6 \mathrm{~mm}$ with periappendiceal inflammation, non-opacification with oral or rectal contrast material with periappendiceal inflammation, or the presence of an appendicolith. In all studies, the diagnostic standard was pathological diagnosis if the patient had an appendectomy or prolonged follow up (usually $3 \mathrm{mo}$ ) if the patient did not have an appendectomy. Studies reported that the sensitivity of CT scanning ranged from $77-100 \%$, the specificity ranged from $83-100 \%$, and the accuracy ranged from $88-98 \%$ for the diagnosis of acute appendicitis in adult men and women.

\section{CONCLUSION}

In adults, the use of computed tomographic scanning has high sensitivity, specificity, and accuracy for diagnosing acute appendicitis.

\section{Commentary}

7 hese 2 systematic reviews provide interesting insights for the clinician who is faced with the dilemma of a patient with suspected appendicitis.

The article by Andersson examines various clinical predictors, including patient demographics, historical features, physical signs, and laboratory investigations to determine their discriminatory power in the patient with suspected appendicitis. A comprehensive systematic review of the literature was done. The studies selected for inclusion involved patients admitted to hospital for suspected appendicitis, and a median $40 \%$ of patients had confirmed appendicitis. Although the generalisability of these findings to other clinical settings with a lower rate of appendicitis may be questioned, some interesting observations are made.

Andersson presents tables with the accuracy and positive and negative likelihood ratios for 28 potential diagnostic variables. Overall, the

For correspondence: Dr L Neumayer, University of Utah, Salt Lake City, UT, USA. Leigh.neumayer@hsc.utah.edu

Source of funding: not stated. individual variables had poor to moderate accuracy and discriminatory ability. It's not surprising that appendicitis remains a challenge to diagnose

All of the individual factors on the history and physical examination had only modest accuracy, with the best being migration of the pain. Signs of peritoneal irritation, including percussion and rebound tenderness, also had moderate accuracy, and the worst overall performer was the rectal examination, which had no discriminatory power.

The white blood cell count and granulocyte count, often maligned for being unhelpful or even misleading in the diagnosis of appendicitis, were 2 of the more accurate predictors of appendicitis, and the positive and negative likelihood ratios became progressively more helpful at more extreme lab values (therefore, the higher the white blood cell count, the more likely the patient had appendicitis). Several studies looked at combinations of variables and found that if several markers of inflammation were abnormal, the likelihood ratios for appendicitis were much higher.

Not surprisingly, no single test or combination of variables could be identified to reliably diagnose, or exclude, appendicitis. Therefore, the formation of an overall clinical impression (or "best guess"), using the history, physical examination and a few lab tests will have to continue (with or without a rectal exam).

However, perhaps a CT scan can be used to settle the issue. Neumayer and Kennedy did a systematic review of studies investigating the accuracy of CT in the diagnosis of appendicitis. This review includes a limited selection of studies from a search of PubMed for English language articles from a short time period of 5.5 years. A single author selected studies for inclusion, and the results of the systematic review are presented in a narrative format, which may be appropriate given the heterogeneous nature of the studies, although this may be more sensitive to bias.

Although no summary statistic was calculated, the sensitivity and specificity of CT scanning for appendicitis was quite high $1>90 \%$ in the majority of the studies) and seemed similar in studies that included atypical presentations only compared with those including all patients. In several studies, the results of the scan frequently changed the intended disposition of the patients, and surgery or hospital admission was avoided or expedited.

The authors conclude that their data support the routine use of CT scanning in the diagnosis of appendicitis. Although CT scans appear to be useful diagnostic tests for appendicitis, several questions remain unanswered. The sensitivity of CT scans for appendicitis is likely $>90 \%$, but whether this sensitivity is high enough to safely exclude appendicitis (and cancel planned surgeries) is unclear, considering the consequences of missed appendicitis. The usefulness of CT scans in patients with a lower suspicion of appendicitis, or in areas where the test is less accessible, remains to be determined. In addition, the role of ultrasound was not addressed (particularly in women, for whom the rate of misdiagnosis is higher and ultrasound more helpful), ${ }^{1}$ and concerns about radiation exposure from liberal use of diagnostic imaging ${ }^{2}$ warrant further study of the best use of this diagnostic test.

Marcia Edmonds, MD University of Alberta Edmonton, Alberta, Canada

1 Flum DR, Morris A, Koepsell T, et al. Has misdiagnosis of appendicitis decreased over time? A population-based analysis. JAMA 2001;286:1748-53.

2 Lee Cl, Haims AH, Monico EP, et al. Diagnostic CT scans: Assessment of patient, physician, and radiologist awareness of radiation dose and possible risks. Radiology 2004;231:393-8. 